13

\title{
Особенности отражения электромагнитных волн СВЧ-диапазона от магнитных коллоидов
}

\author{
(С С.Д. Туркин, Ю.И. Диканский
}

Северо-кавказский федеральный университет, Институт математики и естественных наук, 355009 Ставрополь, Россия

e-mail: dikansky@mail.ru, turkinSD@gmail.com

Поступило в Редакцию 10 апреля 2020 г.

В окончательной редакции 18 мая 2020 г.

Принято к публикации 21 мая 2020 г.

Проведено экспериментальное исследование отражения электромагнитной (ЭМ) волны СВЧ-диапазона на частоте $10.65 \mathrm{GHz}$ от слоя магнитной жидкости (магнитного коллоида) и композитов, синтезированных на ее основе. Установлено, что при повышении концентрации твердой фазы в однородном магнитном коллоиде изменяются условия возникновения ферромагнитного резонанса. Выявлены особенности процесса отражения ЭМ волны от слоя магнитной эмульсии при воздействии магнитных полей, значение напряженности которых гораздо меньше напряженности, соответствующей условию ферромагнитного резонанса. Проведены теоретические расчеты модуля коэффициента отражения от пятислойной системы, содержащей магнитную эмульсию, с последующим сравнением их результатов с экспериментальными данными. В этом же диапазоне напряженностей полей исследовано отражение ЭМ волны от слоя композита магнитной жидкости и немагнитных частиц из бронзы микронного размера.

Ключевые слова: СВЧ, магнитные коллоиды, ферромагнитный резонанс, магнитодиэлектрические эмульсии, магнитное упорядочение, структурная организация.

DOI: 10.21883/JTF.2021.01.50285.125-20

\section{Введение}

В настоящее время происходит интенсивное изучение резонансных свойств ансамбля слабо взаимодействующих магнитных наночастиц [1-3]. Достаточное количество работ посвящено изучению магнитного резонанса образцов в виде порошков, магнитных пленок из ультрадисперсных наночастиц, полученных измельчением моно-, поликристаллов различного химического состава [4-7]. Исследования показывают, что хорошим физическим приближением является модель, приписывающая каждой частице единый магнитный момент. В этом плане представляется интересным изучение, так называемых магнитных наноколлоидов (магнитных жидкостей), в которых ансамбль ультрадисперсных частиц с единым магнитным моментом распределен в немагнитной среде. Взаимодействие таких жидкостей с СВЧ электромагнитными полями также носит резонансный характер, который ранее теоретически рассматривался в работах [8-10]. Ферромагнитный резонанс в таких средах приближенно описывается теорией динамического намагничивания однодоменной частицы во внутреннем поле анизотропии и внешнем магнитном поле. При частоте переменного возбуждающего поля порядка $10 \mathrm{GHz}$ он наблюдается в полях напряженностью $H \sim 10^{5} \mathrm{~A} / \mathrm{m}$ [11-13]. В более слабых полях при этих частотах, как показано в работе [8], динамическая комплексная магнитная восприимчивость магнитных жидкостей теряет резонансный характер. В этом случае при выполнении условия

$$
\bar{\sigma}=\frac{\mu_{0} M_{d} V \bar{H}}{k T} \ll 1,
$$

$\left(M_{d}\right.$ - намагниченность насыщения твердого магнетика, $V$ - объем однодоменной частицы), она описывается выражением, следующим из теории Дебая [14]. В работе [15] установлено, что в такой ситуации отражение ЭМ волн от однородной магнитной жидкости носит анизотропный характер, обусловленный ориентирующим действием внешнего поля на магнитные моменты ферромагнитных частиц. Экспериментальное исследование процессов взаимодействия сверхвысокочастотного излучения с однородной магнитной жидкостью в диапазоне частот от 20 до $40 \mathrm{GHz}$ при воздействии однородных магнитных полей напряженностью до $920 \mathrm{kA} / \mathrm{m}$ ранее проводилось авторами работ [16-19]. Ими установлена зависимость коэффициента отражения СВЧ-излучения от плоского слоя однородной магнитной жидкости от температуры и частоты излучения, позволившая получить информацию о параметрах исследуемых магнитных жидкостей. Обнаруженные минимумы на частотных зависимостях отраженной мощности связаны с дисперсией динамической восприимчивостью наночастиц (с ферромагнитным резонансом) и объяснены на основе теории динамического намагничивания однодоменной частицы во внутреннем поле анизотропии и внешнем намагничивающем поле.

Следует заметить, что на процессы намагничивания магнитных жидкостей значительное влияние оказывают 
взаимодействие коллоидных частиц и связанное с ним структурное состояние системы [20-22]. При этом интенсивность взаимодействия частиц зависит от их концентрации и повышается при ее увеличении. В связи с этим можно предположить, что изменение концентрации дисперсной фазы в однородных магнитных коллоидах может приводить к особенностям отражения от их поверхности СВЧ-излучения и изменению условия возникновения ферромагнитного резонанса. Кроме того, на основе магнитных жидкостей созданы новые композиционные намагничивающиеся среды - магнитные эмульсии [23-26] и магнитные коллоиды с мелкодисперсным наполнителем как из непроводящих, так и проводящих частиц микронного размера [27-31]. Структурой таких сред можно управлять воздействием даже относительно слабых магнитных полей, которое приводит к деформации магнитных капель в магнитных эмульсиях и образованию цепочечной и веретенообразной структуры из немагнитных частиц в коллоидах с немагнитным мелкодисперсным наполнителем. Это должно в свою очередь привести к изменениям характера взаимодействия электромагнитных волн с такими средами. Подтверждением этого могут служить результаты, проведенных нами ранее исследований эффекта Фарадея, наблюдающегося в магнитных коллоидах и созданных на их основе магнитных эмульсиях в СВЧ-диапазоне [32,33]. В связи с выше изложенным в настоящей работе предпринято исследование особенностей процесса отражения ЭМ волны от слоя однородного магнитного коллоида, обусловленных изменением его концентрации в интервале напряженностей поля, соответствующем возникновению ферромагнитного резонанса. Кроме того, исследовано отражение СВЧ-излучения от созданных на основе магнитных жидкостей композитных сред при воздействии постоянного магнитного поля, напряженность которого достаточна для структурного изменения системы, но не превышает значения, при котором возможен ферромагнитный резонанс.

\section{1. Методы исследования}

В отличие от традиционного измерения поглощения с помощью ЭПР-спектрометров экспериментальные исследования в настоящей работе были проведены при использовании установки, аналогичной описанной в [16-19]. Ее схема приведена на рис. 1. Плоскополяризованное излучение (мода $\left.H_{10}\right)$, генерируемое диодом Ганна 1 с частотой $10.65 \mathrm{GHz}$, направлялось по прямоугольному волноводу ( $H$-плечо) в двойной СВЧ тройник, пройдя через аттенюатор 2 и PIN-диод 3. Затем энергия излучения разделялась в опорных плечах $\Pi_{1}$ и $\Pi_{2}$. Левое плечо $\Pi_{1}$ содержало ячейку с образцом 4 и неподвижный короткозамыкающий поршень 5. В правом плече $\left(\Pi_{2}\right)$ был расположен подвижный короткозамыкающий поршень 6 , регулируемый микрометрическим винтом. Отраженные волны от обоих плеч интерфе-

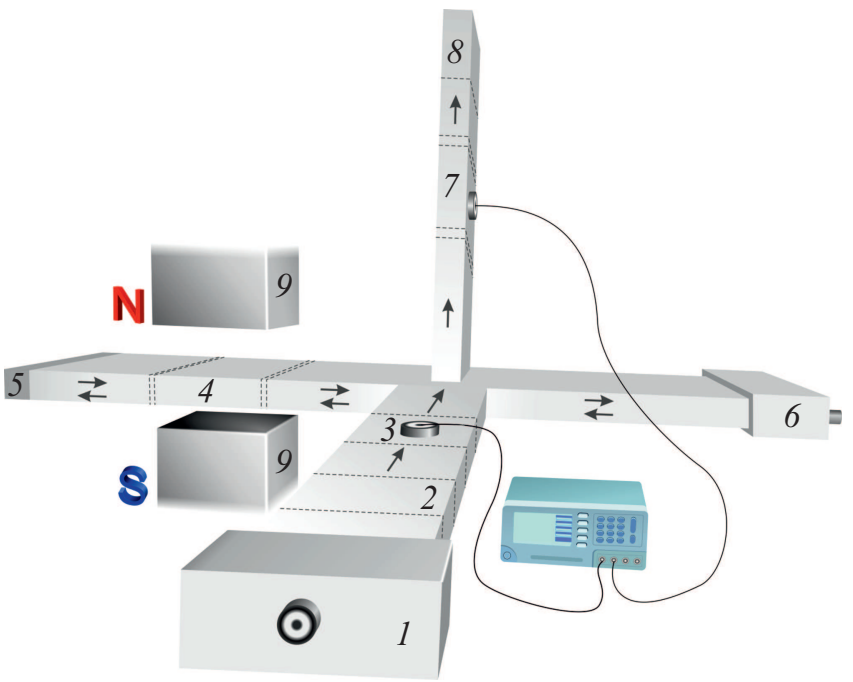

Рис. 1. Схема экспериментальной установки: 1 - генератор сантиметрового излучения, $2-$ фиксированный аттенюатор, 3 - Pin-диод, 4 - кювета с образцом, 5 - неподвижный короткозамыкающий поршень, 6 - подвижный короткозамыкающий поршень, 7 - измерительная линия с квадратичным детектором, 8 - отрезок линии, содержащий поглощающий элемент, 9 - электромагнит, создающий постоянное магнитное поле.

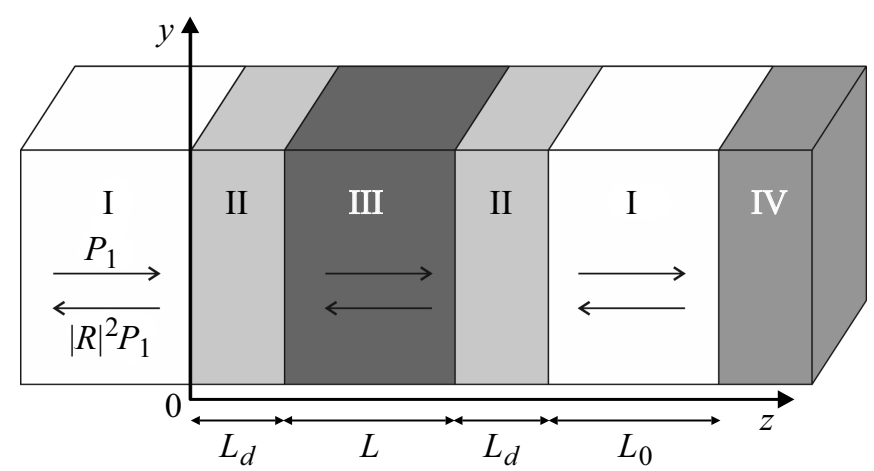

Рис. 2. Подробная схема ячейки с исследуемым образцом: I - пустые области, II - прозрачный для СВЧ излучения диэлектрик, препятствующий растеканию жидкого образца, III - область, заполненная исследуемым образцом, IV неподвижный короткозамыкающий поршень.

рировали в центре двойного тройника, при этом перераспределенная энергия направлялась в вертикальное $E$-плечо. Мощность результирующей волны регистрировалась измерительной линией 7 , представляющей собой отрезок прямоугольного волновода. Последняя была соединена с линией, на торцевой части которой была установлена согласующая нагрузка в виде поглощающей пластины 8 , дающей возможность исключить паразитные отраженные сигналы. Внешнее постоянное однородное магнитное поле $H_{0}$, направленное перпендикулярно вектору распространения волны, создавалось электромагнитом 9. 
На рис. 2 изображена подробная схема ячейки с образцом. Номером I обозначены пустые области. Исследуемый жидкий образец, ограниченный с обеих сторон прозрачным для СВЧ излучения диэлектриком II, содержится в области III. Область IV занимает короткозамыкающий неподвижный поршень. Мощность отраженного излучения характеризуется модулем коэффициента отражения $\tilde{R}$, в общем случае представляющего собой комплексную величину [34,35].

В отсутствие образца зависимость результирующей мощности излучения, поступающей в вертикальное $E$ плечо, от разности хода $\Delta X$ в опорных плечах определяется выражением [34]:

$$
P=P_{0} \sin ^{2}\left(\frac{2 \pi}{\lambda_{\mathrm{B}}}(\Delta X+0.06)\right),
$$

где $P_{0}-$ мощность сигнала, поступающего в двойной тройник от генератора, $\lambda_{\text {в }}$ - длина волны в волноводе:

$$
\lambda_{\mathrm{B}}=\frac{\lambda}{\sqrt{1-\left(\frac{\lambda}{2 a}\right)^{2}}}=2.93 \mathrm{~cm},
$$

$\Delta X$ - разность хода, возникающая за счет различной длины опорных плеч, отсчитываемая от нуля системы координат и регулируемая подвижным короткозамыкающим поршнем; $a=2.3 \mathrm{~cm}$ - размер широкой стенки волновода; 0.06 - постоянная добавочная разность хода, обусловленная конструкцией подвижного поршня.

При наличии образца мощность $P$ поступающего в $E$ плечо излучения описывается уравнением [34]:

$$
P=\frac{P_{0}}{4}\left(1+|\tilde{R}|^{2}+2|\tilde{R}| \cos \left(\frac{4 \pi}{\lambda_{\mathrm{B}}}(\Delta X+0.06)+\pi\right)\right),
$$

где $\pi$ - начальный сдвиг фаз, указывающий на то, что при условии минимума интерференции отраженных волн в опорных плечах вся энергия поступает в вертикальное $E$-плечо. В этом случае измерительная линия регистрирует максимальное значение мощности сигнала, являющегося результатом интерференции волн в опорных плечах.

Последнее уравнение дает возможность рассчитать модуль коэффициента отражения по экспериментально полученным данным. Действительно, функция $P(\Delta X)$ имеет максимальное значение $P_{\max }$, определяемое из эксперимента, при

$$
\cos \left(\pi+\frac{4 \pi}{\lambda_{\mathrm{B}}}(\Delta X+0.06)\right)=1 .
$$

При этом выражение (4) принимает вид стандартного квадратного уравнения

$$
|\tilde{R}|^{2}+2|\tilde{R}|+1-\frac{4 P_{\max }}{P_{0}}=0,
$$

использование которого и позволяет определить модуль коэффициента отражения.
Кроме экспериментальных исследований была предпринята также попытка теоретического анализа особенностей отражения СВЧ излучения от магнитных коллоидных систем. В этом случае для вывода аналитического выражения коэффициента отражения использована система уравнений [35]:

$$
\begin{aligned}
& \left\{\begin{array}{l}
\Delta \tilde{H}+\tilde{k}^{2} \tilde{H}=0, \\
\Delta \tilde{E}+\tilde{k}^{2} \tilde{E}=0,
\end{array}\right. \\
& \tilde{k}=\omega \sqrt{\varepsilon \tilde{\mu} \varepsilon_{0} \mu_{0}},
\end{aligned}
$$

где $\omega-$ циклическая частота колебаний ЭМ поля.

Применительно к пяти областям ячейки комплексные выражения для электрической и магнитной компонент электромагнитной волны имеют вид:

$$
\left\{\begin{array}{l}
\tilde{E}_{\mathrm{I}}=\tilde{E}(x)\left(A_{\mathrm{I}} e^{-\gamma_{0} z}+B_{\mathrm{I}} e^{\gamma_{0} z}\right), \\
\tilde{H}_{\mathrm{I}}=-\gamma_{0} \tilde{H}(x)\left(A_{\mathrm{I}} e^{-\gamma_{0} z}-B_{\mathrm{I}} e^{\gamma_{0} z}\right), \\
\tilde{E}_{\mathrm{II}}=\tilde{E}(x)\left(A_{\mathrm{II}} e^{-\gamma_{d} z}+B_{\mathrm{II}} e^{\gamma_{d} z}\right), \\
\tilde{H}_{\mathrm{II}}=-\gamma_{d} \tilde{H}(x)\left(A_{\mathrm{II}} e^{-\gamma_{d} z}-B_{\mathrm{II}} e^{\gamma_{d} z}\right), \\
\tilde{E}_{\mathrm{III}}=\tilde{E}(x)\left(A_{\mathrm{III}} e^{-\gamma z}+B_{\mathrm{III}} e^{\gamma z}\right), \\
\tilde{H}_{\mathrm{III}}=-\gamma \tilde{H}(x)\left(A_{\mathrm{III}} e^{-\gamma z}-B_{\mathrm{III}} e^{\gamma z}\right), \\
\tilde{E}_{\mathrm{IV}}=\tilde{E}(x)\left(A_{\mathrm{IV}} e^{-\gamma_{d} z}+B_{\mathrm{IV}} e^{\gamma_{d} z}\right), \\
\tilde{H}_{\mathrm{IV}}=-\gamma_{d} \tilde{H}(x)\left(A_{\mathrm{IV}} e^{-\gamma_{d} z}-B_{\mathrm{IV}} e^{\gamma_{d} z}\right), \\
\tilde{E}_{\mathrm{V}}=\tilde{E}(x)\left(A_{\mathrm{V}} e^{-\gamma_{0} z}+B_{\mathrm{V}} e^{\gamma_{0} z}\right), \\
\tilde{H}_{\mathrm{V}}=-\gamma_{0} \tilde{H}(x)\left(A_{\mathrm{V}} e^{-\gamma_{0} z}-B_{\mathrm{V}} e^{\gamma_{0} z}\right),
\end{array}\right.
$$

где $\tilde{E}(x)$ и $\tilde{H}(x)$ - распредение комплексных амплитуд в направлении, перпендикулярном распространению волны; $A_{i}$ и $B_{i}$ - произвольные коэффициенты, включающие множитель [35]; $\gamma_{0}, \gamma_{d}, \gamma$ - постоянные распространения волны в пустом, заполненном диэлектриком с известными значениеми $\varepsilon_{d}$ и $\mu_{d}=1$ и заполненном магнитной жидкостью пространствах соответственно.

Для постоянных $\gamma_{0}, \gamma_{d}, \gamma$ справедливы выражения:

$$
\left\{\begin{array}{l}
\gamma_{0}^{2}=\frac{\pi^{2}}{a^{2}}-\omega^{2} \varepsilon_{0} \mu_{0}, \\
\gamma_{d}^{2}=\frac{\pi^{2}}{a^{2}}-\omega^{2} \varepsilon_{0} \mu_{0} \varepsilon_{d}, \\
\gamma^{2}=\frac{\pi^{2}}{a^{2}}-\omega^{2} \varepsilon_{0} \mu_{0} \varepsilon \tilde{\mu},
\end{array}\right.
$$

где $\varepsilon_{0}, \mu_{0}$ - электрическая и магнитная постоянные, $\varepsilon-$ диэлектрическая проницаемость магнитной жидкости.

Магнитная проницаемость однородной магнитной жидкости является комплексной величиной и определяется формулой

$$
\tilde{\mu}=1+\chi^{\prime}-i \chi^{\prime \prime}
$$

где $\chi^{\prime}, \chi^{\prime \prime}$ - действительная и мнимая части комплексной восприимчивости. Расчет выражений для $\chi^{\prime}$ и $\chi^{\prime \prime}$ 
был проведен в работе [16] при использовании теории Райхера-Шлиомиса [8]:

$$
\begin{gathered}
\chi^{\prime}=\frac{\varphi_{f} M_{s}}{H} L[\delta(T)] \frac{\left(1+n^{2}\right)^{2} \omega_{H}^{4}+\left(n^{2}-1\right) \omega^{2} \omega_{H}^{2}}{\left(1+n^{2}\right)^{2} \omega_{H}^{4}+2\left(n^{2}-1\right) \omega^{2} \omega_{H}^{4}+\omega^{4}}, \\
\chi^{\prime \prime}=\frac{\varphi_{f} M_{s}}{H} L[\delta(T)] \frac{n \omega_{H}^{2}\left(\omega^{2}+\left(1+n^{2}\right) \omega_{H}^{2}\right)}{\left(1+n^{2}\right)^{2} \omega_{H}^{4}+2\left(n^{2}-1\right) \omega^{2} \omega_{H}^{4}+\omega^{4}}, \\
n=\zeta\left[\frac{1}{L[\delta(T)]}-\frac{1}{\delta(T)}\right], \delta(T)=\frac{\mu_{0} M_{s} V}{k T} H,
\end{gathered}
$$

где $\xi=0.1$ - параметр затухания ларморовой прецессии в однодоменной частице, $L[\delta(T)]$ — функиция Ланжевена, $\omega_{H}=g H-$ приведенное магнитное поле, $M_{s}=477.7 \mathrm{kA} / \mathrm{m}$ - намагниченность насыщения магнетита, $\varphi_{f}$ - объемная концентрация твердой фазы магнитной жидкости.

Коэффициент отражения от границы раздела двух сред в области I определяется отношением комплексных амплитуд отраженной и падающей волн [35]:

$$
\tilde{R}=\frac{\tilde{E}_{\mathrm{I}+}(0)}{\tilde{E}_{\mathrm{I}-}(0)}=\frac{\tilde{H}_{\mathrm{I}+}(0)}{\tilde{H}_{\mathrm{I}-}(0)}=\frac{B_{\mathrm{I}}}{A_{\mathrm{I}}} .
$$

Применяя к системе (8) условие непрерывности тангенциальных составляющих $E_{\tau}$ и $H_{\tau}$ на границах, координаты которых показаны на рис. 2 :

$$
\begin{gathered}
z=0, \quad z=L_{d}, \quad z=L_{d}+L, \\
z=2 L_{d}+L, \quad z=2 L_{d}+L+L_{0},
\end{gathered}
$$

и решив систему относительно $\frac{B_{I}}{A_{\mathrm{I}}}$, получим формулу для коэффициента отражения

$\tilde{R}=\frac{B_{\mathrm{I}}}{A_{\mathrm{I}}}=$

$$
\begin{gathered}
\left\{\begin{array}{l}
\left(\tanh \left(\gamma_{0} L_{0}\right)-1\right)\left[\tanh \left(\gamma_{d} L_{d}\right) \tanh (\gamma L) \gamma_{0} \gamma_{d}\left(\gamma_{d}^{2}+\gamma^{2}\right)\right. \\
\left.-\gamma_{d}^{2} \gamma_{0}^{2}\right]+2 \tanh \left(\gamma_{d} L_{d}\right) \gamma_{0}^{2} \gamma_{d} \gamma-2 \gamma_{d}^{3} \gamma \tanh \left(\gamma_{0} L_{0}\right) \\
\times \tanh \left(\gamma_{d} L_{d}\right)+\tanh ^{2}\left(\gamma_{d} L_{d}\right) \gamma \gamma_{0}\left[\gamma \gamma_{0} \tanh (\gamma L)\right. \\
\left.+\gamma_{d}^{2} \tanh \left(\gamma_{0} L_{0}\right)\right]
\end{array}\right\} \\
/\left\{\begin{array}{l}
\left(\tanh \left(\gamma_{0} L_{0}\right)+1\right)\left[\tanh \left(\gamma_{d} L_{d}\right) \tanh (\gamma L) \gamma_{0} \gamma_{d}\right. \\
\left.\times\left(\gamma_{d}^{2}+\gamma^{2}\right)+\gamma_{d}^{2} \gamma_{0}^{2}\right]+2 \tanh \left(\gamma_{d} L_{d}\right) \gamma_{0}^{2} \gamma_{d} \gamma+2 \gamma_{d}^{3} \gamma \\
\times \tanh \left(\gamma_{0} L_{0}\right) \tanh \left(\gamma_{d} L_{d}\right)+\gamma \gamma_{0} \gamma_{d}^{2}\left[\tanh \left(\gamma_{0} L_{0}\right)\right. \\
\left.+\tanh \left(\gamma_{d} L_{d}\right)+1\right]+\gamma_{d}^{4} \tanh \left(\gamma_{0} L_{0}\right) \tanh { }^{2}\left(\gamma_{d} L_{d}\right) \\
\times \tanh (\gamma L)
\end{array}\right\} .
\end{gathered}
$$

\section{2. Образцы для исследований}

Для проведения исследований были использованы 3 образца, описание которых приведено ниже. Образец № 1 представлял собой магнитный коллоид на основе керосина с магнетитовыми однодоменными частицами, стабилизированными олеиновой кислотой. Средний диаметр частиц составлял $8 \mathrm{~nm}$, исходная объемная концентрация дисперсной фазы - 17.6\%, статическая магнитная проницаемость $\mu_{s t}=7.2$. Образец, как можно судить по наблюдениям в оптический микроскоп, представлял собой однородную среду без видимых включений и агрегатов из коллоидных частиц.

Образец № 2 был получен диспергированием образца № 1 с исходной объемной концентрацией дисперсной фазы $(17.6 \%)$ в несмешивающемся с ним масле АМГ-10 и представлял собой эмульсию с объемной долей магнитных капель $30 \%$. Средний диаметр капель составлял $2 \mu \mathrm{m}$, коэффициент поверхностного натяжения границы капли со средой $-2 \cdot 10^{-6} \mathrm{H} / \mathrm{m}$.

Образец № 3 представлял собой композит однородной магнитной жидкости (с концентрацией дисперсной фазы $12 \%$ ) и немагнитных частиц из бронзы микронного размера. Статическая магнитная проницаемость использовавшейся магнитной жидкости для получения образца $\mu_{s t}=4$, средний диаметр ее коллоидных частиц состалял $8 \mathrm{~nm}$. Исследования проводились при концентрации частиц наполнителя 9 и 17\%, для сравнения получали также соответствующие зависимости для исходного образца магнитной жидкости не содержащего какихлибо включений. Для дополнительной характеристики образца № 3 отметим, что введение немагнитных частиц („магнитных дырок“) в магнитную жидкость эквивалентно образованию в ней частиц, имеющих намагниченность, направленную противоположно намагниченности окружающей среды [36]. Вследствие этого воздействие даже относительно слабого магнитного поля на такую среду приводит к объединению немагнитных частиц наполнителя в цепочечные структуры, что и наблюдается для данного образца.

\section{3. Экспериментальные результаты и их обсуждение}

Установлено, что зависимость мощности сигнала, отраженного от поверхностей слоя однородного образца магнитной жидкости, от напряженности внешнего магнитного поля, направленного перпендикулярно вектору распространения волны, претерпевает минимум в области значений напряженностей, соответствующих условию ферромагнитного резонанса. В качестве подтверждения этого на рис. 3 изображена зависимость максимальной регистрируемой мощности от напряженности приложенного постоянного магнитного поля. Как видно из рисунка, зависимость претерпевает минимум в окрестности значений $H_{0}$, соответствующих ферромагнитному резонансу $\left(P^{\prime}-\right.$ регистрируемая максимальная мощность при $\left.H_{0}=0\right)$. Очевидно, его наличие связано с резонансным поглощением энергии электромагнитного поля в исследуемой среде.

На рис. 4 представлены зависимости разности хода (возникающей за счет различной длины опорных плеч), 


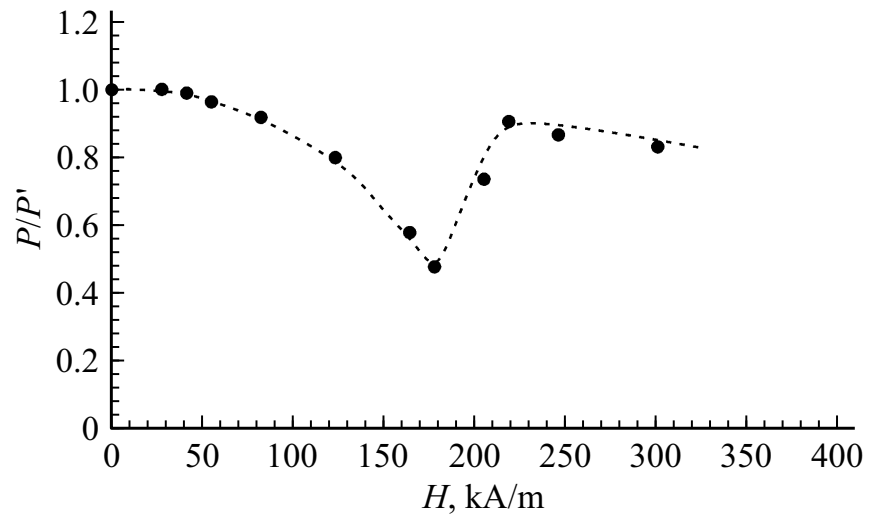

Рис. 3. Относительное изменение максимальной регистрируемой мощности от внешнего постоянного магнитного поля $\left(P^{\prime}-\right.$ регистрируемая максимальная мощность при $\left.H_{0}=0\right)$.

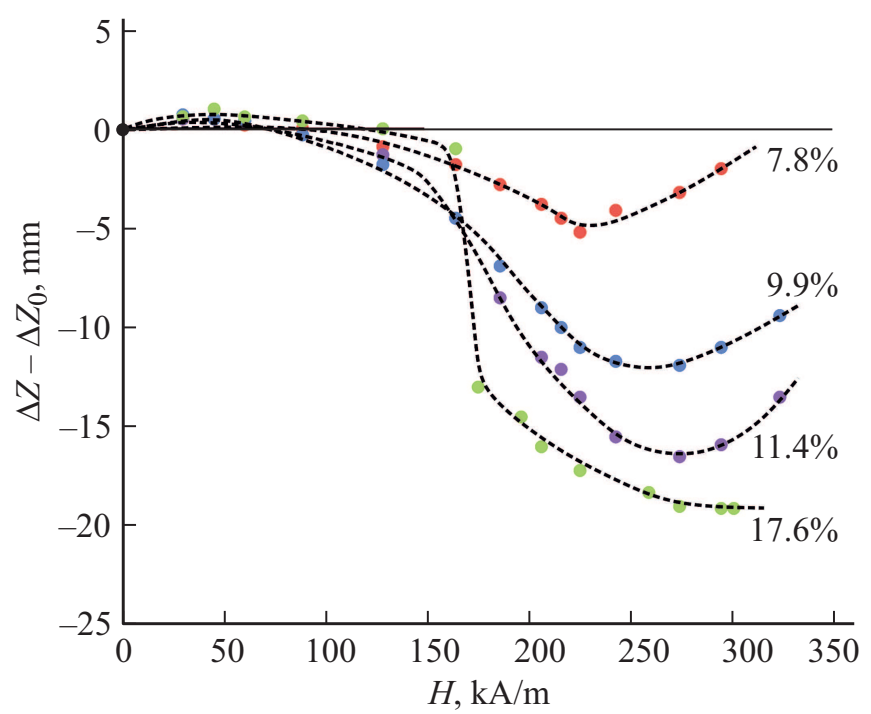

Рис. 4. Графики зависимостей разности хода волн в опорных плечах, соответствующих минимальному значению регистрируемой мощности, от напряженности внешнего магнитного поля $H$, полученные при исследовании образцов с различной концентрацией дисперсной фазы $\left(\Delta Z_{0}-\right.$ разность хода при $\left.H_{0}=0\right)$.

соответствующей минимальному значению регистрируемой мощности, от напряженности внешнего магнитного поля, полученные при исследовании образцов с различной концентрацией дисперсной фазы $\left(\Delta Z_{0}-\right.$ разность хода при $\left.H_{0}=0\right)$. Как видно из рисунка, минимумы представленых зависимостей смещаются в область более высоких значений напряженности поля при увеличении концентрации твердого магнетика. Очевидно, что обнаруженные особенности хода этих зависимостей связаны с изменением условий возникновения ферромагнитного резонанса. Учитывая, что повышение концентрации коллоидных частиц приводит к усилению их взаимодействия, можно сделать вывод о существенном влиянии взаимодействия однодоменных частиц на условия возникновения ферромагнитного резонанса в магнитных коллоидах.

Следует также обратить внимание на графики зависимости мощности регистрируемого излучения от разности хода волны в опорных плечах при различных значениях напряженности внешнего магнитного поля (рис. 5). При достижении некоторой величины $H_{0}$ в системе устанавливается так называемый режим согласованного опорного плеча, иллюстрацией которого является кривая 3. В этой ситуации уровень энергии, поступающей в вертикальное $E$-плечо, постоянный, т.е. практически не зависит от разности хода $\Delta Z$ (коэффициент бегущей волны > 0.85). Таким образом, ячейка с магнитной жидкостью в этом случае выступает в качестве регулируемого внешним магнитным полем переменного согласующего элемента. Отметим, что данная ситуация может иметь практическое приминение в измерительной технике СВЧ-устройств.

При исследовании образца № 2 было обнаружено, что коэффициент отражения СВЧ-излучения от его слоя претерпевает существенные изменения при воздействии даже относительно слабых магнитных полей, напряженности которых значительно меньше напряженностей, соответствующих возникновению ферромагнитного резонанса. На рис. 6 представлена экспериментально полученная зависимость (в виде точек) коэффициента отражения образца № 2 от напряженности постоянного магнитного поля, направленного вдоль поверхности слоя. По-видимому, характер полученной зависимости обусловлен влиянием деформации микрокапель магнитной эмульсии, наблюдающейся даже при небольших напряженностях воздействующего постоянного поля. На рис. 7 схематически изображено взаимное расположение вертикально вытянутых капель в постоянном магнитном поле напряженностью $H_{0}$ и вектора магнитного поля электромагнитной волны типа $H_{10}$. Как видно из рисунка, направление напряженности поля электромагнитной волны перпендикулярно большой полуоси вытянутого эллипсоида. Это означает, что деформация капель под

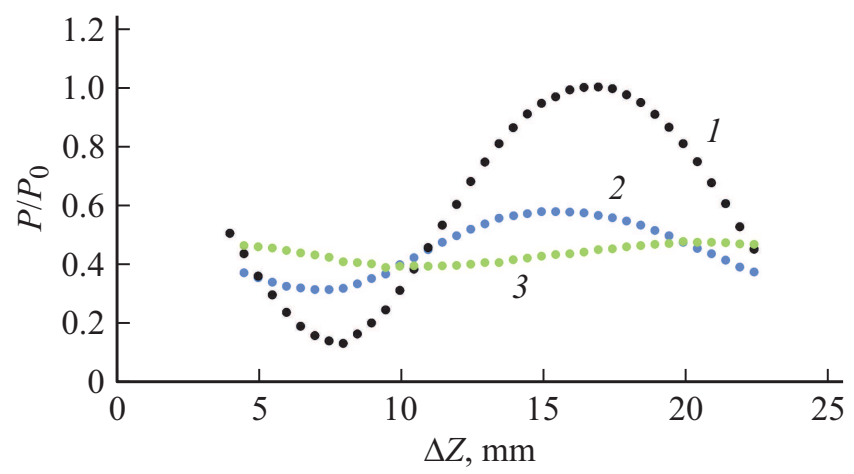

Рис. 5. Относительное изменение регистрируемой мощности излучения от разности хода волн в опорных плечах двойного тройника при различных значения напряженности внешнего магнитного поля (образец № 1). Кривые: $1-0,2-164,3-$ $196 \mathrm{kA} / \mathrm{m}$. 


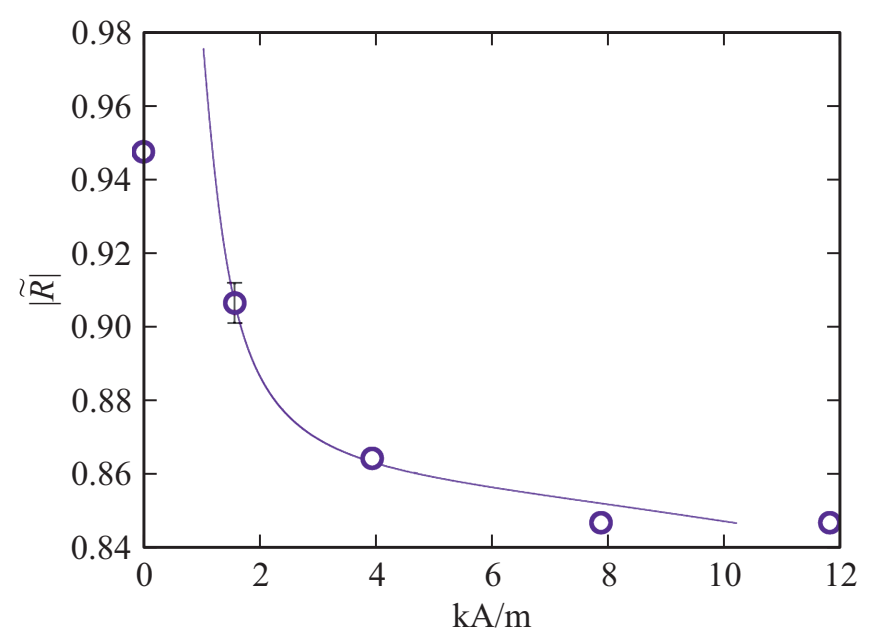

Рис. 6. Зависимость модуля коэффициента отражения образца № 2 (магнитная эмульсия) от внешнего постоянного магнитного поля. Экспериментальная зависимость обозначена в виде точек, теоретически рассчитанная - кривой линией.

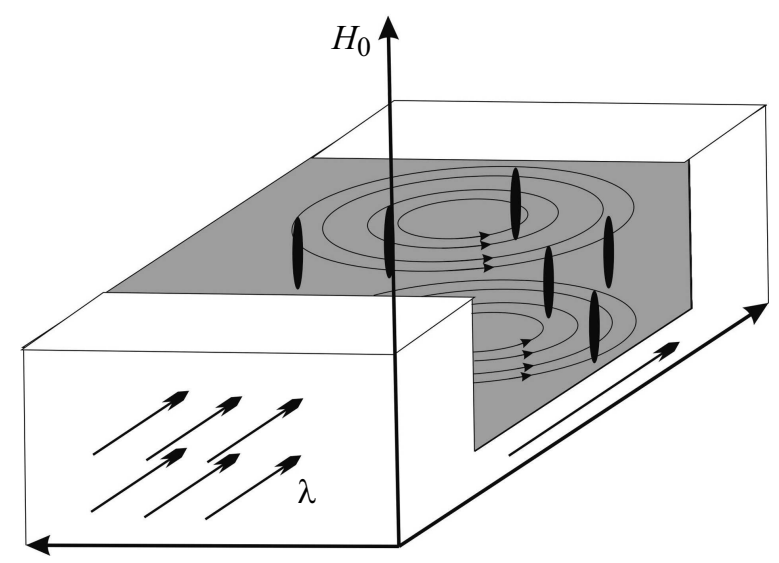

Рис. 7. Схематическое изображение магнитных капель эмульсии вытянутых вдоль вертикального внешнего магнитного поля $H_{0}$ относительно магнитного поля волны $H_{10}$.

воздействием постоянного поля приводит к увеличению размагничивающего фактора для магнитного поля электромагнитной волны. Деполяризующий фактор для электрической компоненты, наоборот, уменьшается, вследствие сонаправленности вектора напряженности электрического поля волны с большой полуосью вытянутого эллипсоида.

Для теоретического расчета коэффициента отражения $\tilde{R}$ определим выражения для магнитной и диэлектрической проницаемостей эмульсии. Отметим, что длина волны СВЧ-излучения, использованного в экспериментальных исследованиях, гораздо больше размера капель магнитной эмульсии $\left(\lambda \gg R_{0}\right)$. В таком случае возможно использование приближения сплошной среды, при этом магнитная $\tilde{\mu}$ и диэлектрическая $\varepsilon$ проницаемости для сплошных сред в системе (9), должны быть заменены на эффективные проницаемости магнитной эмульсии (модель эффективной среды [36]):

$$
\begin{gathered}
\tilde{\mu}_{\mathrm{eff}}=\mu_{e}\left(1+\frac{\left(\tilde{\mu}-\mu_{e}\right) \varphi}{\mu_{e}+N_{1}\left(\tilde{\mu}-\mu_{e}\right)(1-\varphi)}\right), \\
\varepsilon_{\mathrm{eff}}=\varepsilon_{e}\left(1+\frac{\left(\varepsilon-\varepsilon_{e}\right) \phi}{\varepsilon_{e}+N_{2}\left(\varepsilon-\varepsilon_{e}\right)(1-\phi)}\right),
\end{gathered}
$$

где $\mu_{\mathrm{B}}$ и $\varepsilon_{\mathrm{B}}-$ магнитная и диэлектрическая проницаемости немагнитной среды АМГ-10 $\left(\mu_{\mathrm{B}}=1\right), \varphi=30 \%-$ концентрация эмульсии, $\varepsilon$ и $\tilde{\mu}-$ диэлектрическая и магнитная проницаемости магнитной жидкости на данной частоте, $N_{1}$ - размагничивающий фактор вдоль малой оси вытянутой капли, $N_{2}$ - деполяризующий фактор вдоль его большой оси.

Для деполяризующего фактора вдоль длинной оси эллипсоидальной капли используем известное выражение [24]:

$$
N_{2}=\frac{1-e^{2}}{2 e^{3}}\left(\ln \frac{1+e}{1-e}-2 e\right),
$$

где $e-$ эксцентриситет эллипсоида.

Поскольку численные значения для размагничивающего и деполяризующего факторов вдоль одной и той же оси эллипсоида одинаковы, то размагничивающий фактор вдоль направления напряженности магнитного поля волны может быть определен как:

$$
N_{1}=\frac{1-N_{2}}{2} \text {. }
$$

Следует отметить, что деполяризующий фактор $N_{2}$ численно равен размагничивающему фактору вдоль направления внешнего постоянного поля $N_{H}$. Зависимость последнего от напряженности внешнего магнитного поля, приводящего к деформации капли, может быть определена выражением из работы [36]:

$$
H_{0}^{2}=\frac{2 \sigma}{\mu_{0} R_{0}}\left(\frac{1}{\mu_{s t}-1}+N_{H}\right)^{2} \frac{\frac{3-2 e^{2}}{e^{2}}-\frac{\left(3-4 e^{2}\right) \arcsin (e)}{\left(e^{3} \sqrt{1-e^{2}}\right)}}{\left(1-e^{2}\right)^{2 / 3}\left[\frac{3-e^{2}}{e^{5}} \ln \frac{1+e}{1-e}-\frac{6}{e^{4}}\right]},
$$

где $\sigma=2 \cdot 10^{-6} \mathrm{~N} / \mathrm{m}$ - коэффициент поверхностного натяжения границы капли со средой, $\mu_{s t}-$ статическая магнитная проницаемость магнитной жидкости, являющаяся постоянной величиной в области малых значений вектора напряженности внешнего магнитного поля, $R_{0}=2 \cdot 10^{-6} \mathrm{~m}$ - радиус невозмущенной капли.

Таким образом, с учетом того что $N_{2}=N_{H}$, последнее выражение и выражение (18) для $N_{2}$ позволяют численными методами определить диапазон значений эксцентриситета, соответствующих интервалу значений напряженности деформирующего каплю магнитного поля. Это в свою очередь дает возможность определить при этих напряженностях значения эффективной магнитной проницаемости эмульсии, входящей в постоянную распространения $\gamma$ СВЧ волны, проходящей через образец и, в конечном итоге, рассчитать зависимость 


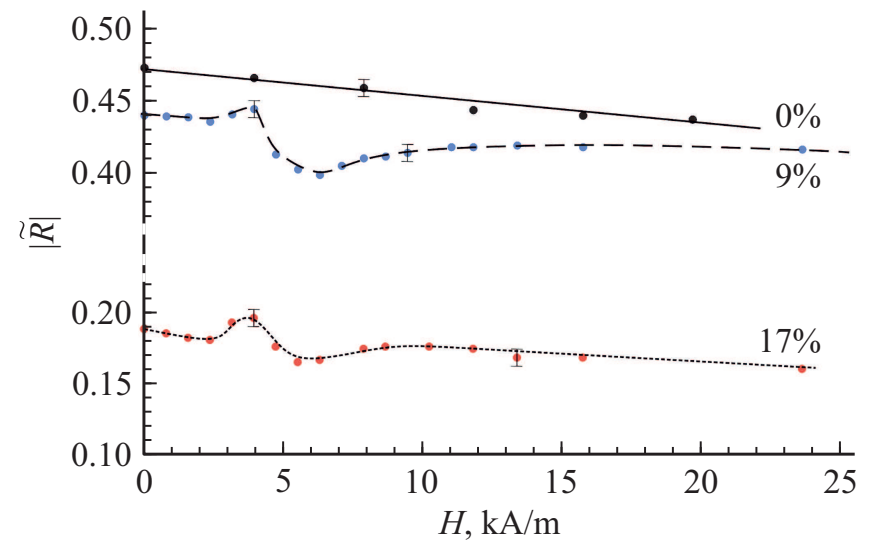

Рис. 8. График зависимости модуля коэффициента отражения образца № 3 от напряженности внешнего постоянного магнитного поля при различных концентрациях немагнитных проводящих частиц. Концентрация $0 \%$ соответствует исходному однородному образцу.

коэффициента отражения от напряженности внешнего магнитного поля.

На рис. 6 кроме экспериментальной зависимости коэффициента отражения от напряженности внешнего постоянного магнитного поля (кривая в виде точек) приведена также аналогичная зависимость, рассчитанная теоретически (гладкая кривая) по формуле (15). Их качественное согласие позволяет предположить, что резкий спад коэффициента отражения в области малых полей действительно связан с наличием и деформацией, под воздействием поля, капель эмульсии.

При исследовании образца № 3 в области малых значений напряженности внешнего магнитного поля было обнаружено, что наличие в коллоиде проводящих немагнитных частиц бронзы микронного размера приводит к существенному изменению зависимости коэффициента отражения от напряженности внешнего магнитного поля. На рис. 8 представлен график зависимостей модуля комплексного коэффициента отражения от напряженности внешнего магнитного поля при различных концентрациях немагнитных проводящих частиц (концентрация 0\% соответствует исходному однородному образцу). Как видно из рисунка, в случае однородного коллоида график зависимости представляет собой монотонно слабо убывающую зависимость, что согласуется с выражениями для восриимчивости (11) и (12). При добавлении проводящих частиц характер этой зависимости изменяется, при этом в области напряженностей 4-6kA/m наблюдаются экстремумы, не характерные для однородного образца. Можно предположить, что это связано с рассмотренным выше возникновением в этом образце упорядоченной структуры и из немагнитных микронных частиц (объединением их в агрегаты цепочечного вида). Следует также обратить внимание, что при увеличении объемной концентрации проводящего наполнителя численное значение модуля коэффициента отражения существенно уменьшается, что приводит к понижению значения отраженной энергии от образца. Очевидно, это связано с тем, что добавление проводящих частиц повышает проводимость образца $\xi$. В результате этого увеличивается мнимая компонента комплексной диэлектрической проницаемости

$$
\varepsilon^{*}=\frac{\xi}{\omega \varepsilon_{0}},
$$

что приводит к более интенсивному затуханию электрической компоненты электромагнитной волны в образце.

\section{Заключение}

Таким образом, проведенные исследования выявили ряд особенностей взаимодействия СВЧ излучения с магнитными жидкостями и их композитами. Установлено, что при повышении концентрации твердой фазы в однородной магнитной жидкости изменяются условия возникновения ферромагнитного резонанса, что связано с усилением магнитодипольного взаимодействия коллоидных частиц. В результате этого происходит увеличение значений напряженности внешнего магнитного поля, соответствующих условию возникновения ферромагнитного резонанса.

Показано также, что в области слабых полей, напряженности которых много меньше напряженности, соответствующей возникновению ферромагнитного резонанса, могут происходить существенные изменения коэффициента отражения от композитов магнитной жидкости за счет пространственного упорядочения их структуры. Так, в магнитных эмульсиях такое структурное упорядочение может возникать за счет деформации взвешенных в немагнитной среде капель магнитной жидкости и их вытягивании вдоль направления внешнего магнитного поля. В композите однородной магнитной жидкости и микронных проводящих частиц изменение коэффициента отражения при воздействии поля связано со структурным упорядочением частиц наполнителя. При этом характер зависимости коэффициента отражения от напряженности магнитного поля для такого образца имеет существенные отличия от аналогичной зависимости, полученной при исследовании однородного коллоида. Предполагается, что это связано с объединением проводящих частиц в цепочки, ориентированные вдоль линий вектора напряженности внешнего магнитного поля и параллельного ему вектора напряженности электрического поля волны. В общем случае наличие проводящих частиц существенно понижает значение отраженной энергии электромагнитной волны от образца. Вероятно, это связано с увеличением мнимой компоненты комплексной диэлектрической проницаемости, обусловленное повышением проводимости образца. 


\section{Финансирование работы}

Работа выполнена при финансовой поддержке: Российского фонда фундаментальных исследований: проект № 19-32-90228; Министерства науки и высшего образования РФ в рамках базовой части государственного задания вузам в сфере научной деятельности (проект 0795-2020-0030).

\section{Конфликт интересов}

Авторы заявляют, что у них нет конфликта интересов.

\section{Список литературы}

[1] P.E. Junnson. Adv. Chem. Phys., 128, 191 (2004).

[2] O. Petracie, X. Chen, S. Bedanta, W. Kleeman, S. Sahoo, S. Cardoso, P.P. Freitas. J. Magn. Magn. Mater., 300, 192 (2006).

[3] А.В. Горобинский. Ферромагнитный резонанс в композиционных магнитных наноструктурах. Диссертация на соискание ученой степени кандидата физ.-мат. наук. (Краснодар, 2011)

[4] Yu.A. Koksharov, D.A. Pankratov, S.P. Gubin, I.D. Kossobudsky, M. Beltran, Y. Khodorkovsky, A.M. Tishin. J. Appl. Phys., 89, 2293 (2001). DOI: 10.1063/1.1332417

[5] С.А. Вызулин, Ю.Е. Калинин, Г.Ф. Копытов. Известия высших учебных заведений. Физика. 49 (3), 40 (2006).

[6] С.П. Губин, Ю.А. Кокшаров, Г.Б. Хомутов. Успехи химии, 74 (6), 540-573 (2005).

[7] С.А. Вызулин, А.В. Горобинский, Е.В. Лебедева, Н.Е. Сырьев. Исследование многослойных наноструктурных пленок методом ферромагнитного резонанса. Сборник научных трудов IV Всероссийской конференции ,физические свойства металлов и сплавов“. (Екатеринбург: УГТУ-УПИ, 2007).

[8] Ю.Л. Райхер, В.А. Игнатченко, М. И. Шлиомис. ЖЭТФ, 70, 1300 (1976). [R.S. Gekht, V.A. Ignatchenko, Yu.L. Ralkher, M.I. Shliomis. JETP, 43 (4), 677, (1976).]

[9] Ю.Л. Райхер, М.И. Шлиомис. ЖЭТФ, 67, 1060 (1974) [Yu.L. Ralkher, M.I. Shliomis. JETP, 40, 526 (1975).]

[10] В.А. Игнатченко, Р.С. Гехт. ЖЭТФ, 67, 1506 (1974).

[11] J.C. Anderson, B. Donovan. Proc. Phys. Soc., 73, 593 (1959).

[12] J.C. Anderson, B. Donovan. Proc. Phys. Soc., 75, 33 (1960).

[13] J.C. Anderson, B. Donovan. Proc. Phys. Soc., 75, 149 (1960).

[14] P. Debye. Polar Molecules (Chemical Catalog Company, NY., 1929).

[15] Д.А. Усанов, Ал.В. Скрипаль, Ан.В. Скрипаль, С.А. Ермолаев. Письма в ЖТФ, 18 (23), 44 (1992). [D.A. Usanov, Al.V. Skripal', An.V. Skripal', S.A. Ermolaev, Tech. Phys. Lett., 18 (12), 780 (1992).]

[16] Д.А. Усанов, А.Э. Постельга, С.В. Алтынбаев. ЖТФ, 83 (11), 30 (2013). [D.A. Usanov, A.E. Postelga, S.V. Altynbaev. Tech. Phys., 58, 1578 (2013). DOI: $10.1134 / \mathrm{S} 1063784213110273]$

[17] Д.А. Усанов, Ал.В. Скрипаль, Ан.В. Скрипаль, А.Э. Постельга. ЖТФ, 79(9), 146 (2009). [D.A. Usanov, Al.V. Skripal', An.V. Skripal', A.E. Postelga. Tech. Phys., 54, 1389 (2009). DOI: 10.1134/S1063784209090229]
[18] Д.А. Усанов, Ал.В. Скрипаль, Ан.В. Скрипаль, А.В. Курганов. ЖТФ, 71 (12), 26 (2001). [D.A. Usanov, Al.V. Skripal', An.V. Skripal', A.V. Kurganov. Tech. Phys., 46, 1514 (2001). DOI: $10.1134 / 1.1427984]$

[19] Д.А. Усанов, Ал.В. Скрипаль, Ан.В. Скрипаль, А.Э. Постельга, Ю.Л. Райхер, В.И. Степанов. ЖТФ, 76 (11), 126 (2006). [D.A. Usanov, Al.V. Skripal', An.V. Skripal', A.E. Postelga, Yu.L. Raikher, V.I. Stepanov. Tech. Phys., 51, 1520 (2006). DOI: 10.1134/S1063784206110211]

[20] Ю.И. Диканский. Эффекты взаимодействия частич и структурно кинетические процессы в магнитных коллоидах. Диссертация на соискание ученой степени доктора физ.-мат. наук. (Ставрополь, 1999).

[21] A.O. Ivanov, O.B. Kuznetsova. Phys. Rev. E, 64 (4), 41405 (2001). DOI: 10.1103/PhysRevE.64.041405

[22] C. Holm, A. Ivanov, S. Kantorovich, E. Pyanzina, E. Reznikov. J. Phys.: Condens. Matter, 18, 2737 (2006).

[23] Y.I. Dikansky, M.A. Bedzhanyan, V.V. Kiselev. Magnetohydrodynamics, 1, 19 (1995).

[24] A. Zakinyan, Y. Dikansky. Colloids and Surfaces, 380, 314 (2011).

[25] M. Ivey, J. Liu, Y. Zhu, S. Cutillas. Phys. Rev. E, 63, 011403 (2000). DOI: 10.1103/PhysRevE.63.011403

[26] A. Zakinyan, Y. Dikansky, M. Bedzhanyan. J. Dispersion. Sci. Technol., 35, 111 (2014). DOI: 10.1080/01932691.2013. 769109

[27] X. Li, X.-D Niu, Y. Li, M.-F. Chen. Phys. Fluid., 30, 040905 (2018). DOI: $10.1063 / 1.5010292$

[28] J. Černák, G. Helgesen, A.T. Skjeltorp. Phys. Rev. E, 70, 031504 (2004). DOI: 10.1103/PhysRevE.70.031504

[29] B.E. Kashevskii, V.I. Kordonskii, I.V. Prohorov. Magnetohydrodynamics, 24 (3), 121 (1998).

[30] J. Popplewell, R.E. Rosensweig. J. Phys. D: Appl. Phys., 29, 2297 (1996).

[31] Yu.I. Dikansky, J.G.Veguera, V.N. Suzdalev, Yu.L. Smerek. Magnetohydrodynamics, 38 (3), 281 (2002).

[32] Ю.И. Диканский, С.Д. Туркин. ЖТФ, 87 (12), 1895 (2017). [Y.I. Dikanskii, S.D. Turkin. Tech. Phys., 62, 1893 (2017). DOI: $10.1134 / \mathrm{S} 1063784217120064]$

[33] S.D. Turkin, Yu.I. Dikansky. Magnetohydrodynamics, 54 (1-2), 3 (2018).

[34] И.В. Лебедев. Техника и приборы СВЧ. (Высшая школа, М., 1970) т. 1-2.

[35] В.В. Никольский. Теория электромагнитного поля. (Высшая школа, М., 1961)

[36] Э.Я. Блум, М.М. Майоров, А.О. Цеберс. Магнитные жсидкости. (Зинатне, Рига, 1986). 